\title{
An Eyelid Detection Algorithm for the Iris Recognition
}

\author{
Pengfei Cai and Chongke Wang \\ Henan Mechanical and Electrical Engineering College, Xinxiang 453002, China \\ cpf_2000@126.com
}

\begin{abstract}
To reduce the influence of the eyelid for the iris recognition rate, an eyelid detection algorithm for the iris recognition is proposed. The grayscale morphological operations are employed to remove the interference of the eyelash and the light spot to the eyelid region. The points of the minimum grayscale value of each column in the eyelid region are extracted as edge points. The least squares parabolic fitting eyelids is carried out for the edge points of the eyelid. The eyelid parabolic moving up and down in neighborhood region is used to localizing the eyelid precisely, when the grayscale mutation happens. The experimental results show that the eyelid localization algorithm can detect the eyelid effectively and quickly. Compared with the hough transform algorithm, the accurate rate is the same, but the speed is improved about 4 seconds.
\end{abstract}

Keywords: eyelid localization, morphological algorithm, least square fitting method

\section{Introduction}

After the iris localization, the annular region segmented contains not only an ideal complete iris texture, in most cases, but also the interference of eyelid, eyelash and spot and the other non-iris region, As shown in Figure 1. If the interference regions cannot be detected and removed, in the subsequent normalization operation and iris matching, the regions will be considered as a part of the iris texture to encode and compare to affect the iris matching accuracy [1].

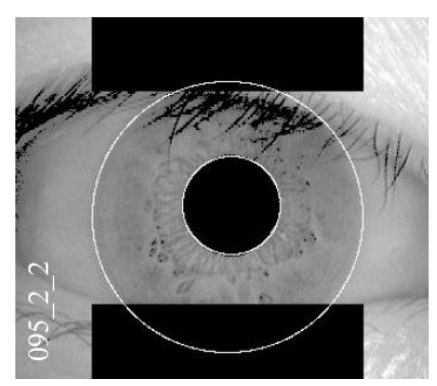

Figure 1. Eyelid and Eyelash Detection

The eyelids are usually modeled as the parabola, and then the optimal target curve is searched in the eye image for the detection. At present, the eyelid detection algorithms mainly include:

(1)the parabola detection method combing the edge detection with the hough space transform [2].

(2)the grayscale integral and differential detection method based on the parabolic path [3];

(3)the parabola fitting based on the least square method [4].

The first kind of method can maximize to extract the iris texture, but the search of three-dimensional space is slow; the second kind of method uses as daugman's classic 
round integral/differential operator localization, because the three-dimensional space search leads to the problem of the slow speed; the third kind of method extracts eyelid edge points, and then uses the least squares for fitting eyelid, and compared to the former two methods, it has lower accuracy, but in one-dimensional space, it can search at the fast speed [5].

Because of the limitations of the existing eyelid detection algorithms, a new kind of eyelid detection algorithm for iris recognition is put forward. First of all, the grayscale morphological operations are used to remove the influence from the eyelash and eyelid interference in the eyelid region; then, the edge points are extracted according to the grayscale characteristics of the eyelid edge, and the least square method is used for fitting to the edges of the eyelids; finally, the edge of the eyelid is located precisely. The experimental results show that the algorithm of the eyelid can detect the eyelid accurately and fast.

\section{The Rough Detection of the Eyelid}

It is often seen that the upper eyelid shades the iris image region. If the shaded area is more than half of the iris region, the iris region is very difficult to identify. The detection steps are divided into segmenting eyelid region and pre-processing, finding the edge point set and eyelid edge fitting.

\subsection{Segmenting Eyelid Region and Pre-processing}

The iris image involves a large part of the eyelid region. To improve the speed of detection, the iris localization algorithm [12] is used to localize the iris, and the results are shown in Figure 2.

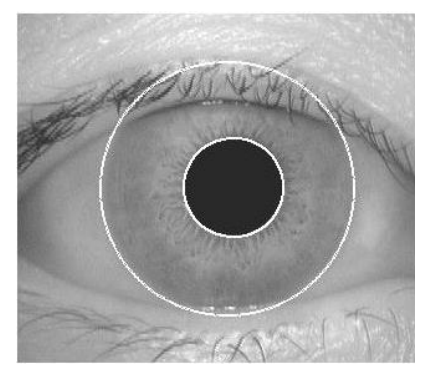

Figure 2. Iris Localization

The eyelid edge region is segmented according to the eyelid segmentation algorithm in the literature [6] and the parameters obtained of the inner and outer edges of iris. To prevent the pupil from being split out, the eyelid region segmented in Figure 2 is shown in Figure 3. The process of the following eyelid detection is carried out in this region.

Because of the influence of the eyelash and small light spot, $5 \times 5$ element's values are selected for disc grayscale morphology structure element. The segmentation of grayscale morphological closing-opening operation [7] is done for the edge of the eyelid region. A grayscale morphological closing operation is carried out for the eyelash area to eliminate the effect of eyelashes and other dark details, and keep relatively bright areas from being affected. Closing morphological operation results are shown in Figure 4. It can be seen that a single eyelash has been eliminated, and the aggregation eyelash region is greatly reduced. A gray level morphological opening operation is carried out for Figure 5, to eliminate the influence of the bright light spot and other details, and relatively keep bright areas and large overall gray level invariant. After the morphological closing-opening operation, the interference of eyelash and light spot is eliminated, and the whole eyelid edge is preserved. The results are shown in Figure 5. 


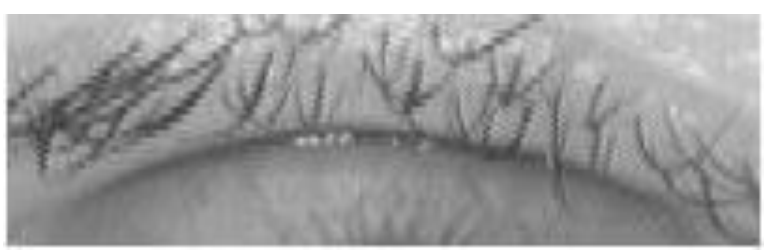

Figure 3. Upper Eyelid Area

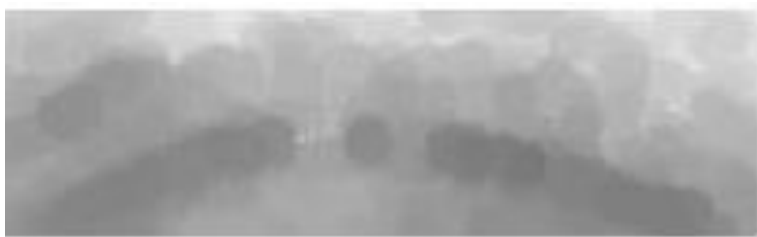

Figure 4. Morphology Close Operation Result

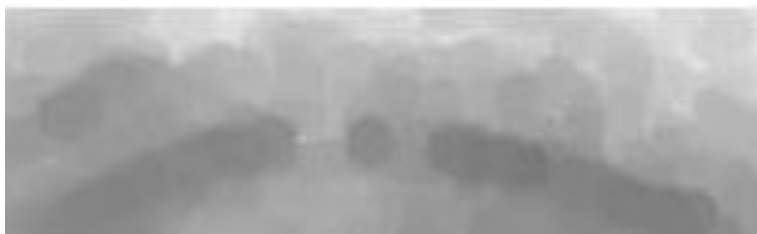

Figure 5. Morphology Close-open Operation Result

\subsection{Searching for the Edge Point Set of Eyelid}

The each column grayscale value of the eyelid margin area is analyzed, which is shown in Figure 6. A total of 8 columns of gray distribution are displayed in the eyelid edge region. The abscissa is the line coordinate, and the ordinate is the grayscale value, with 20 columns per column spacing from left to right[9]. In the Figure 6, each column grayscale distribution has "Valley" point, which is corresponding to the edge of the eyelid. The eyelid edge points on the left and the right ends is the lowest in the edge of the eyelid area, which shows a minimum grayscale value in the distribution map.

According to the above analysis, the eyelid edge points $\left(x_{t}, y_{t}\right)$ satisfy the following conditions:

$$
x_{t}=\arg \min _{x} I\left(x, y_{t}\right)
$$

$I$ is the grayscale function, and $\arg \min _{x}(\exp r(x))$ represents $x$ which makes the formula take the minimum value. The formula means that the eyelid edge points are the corresponding points of each column gray minimum value. 

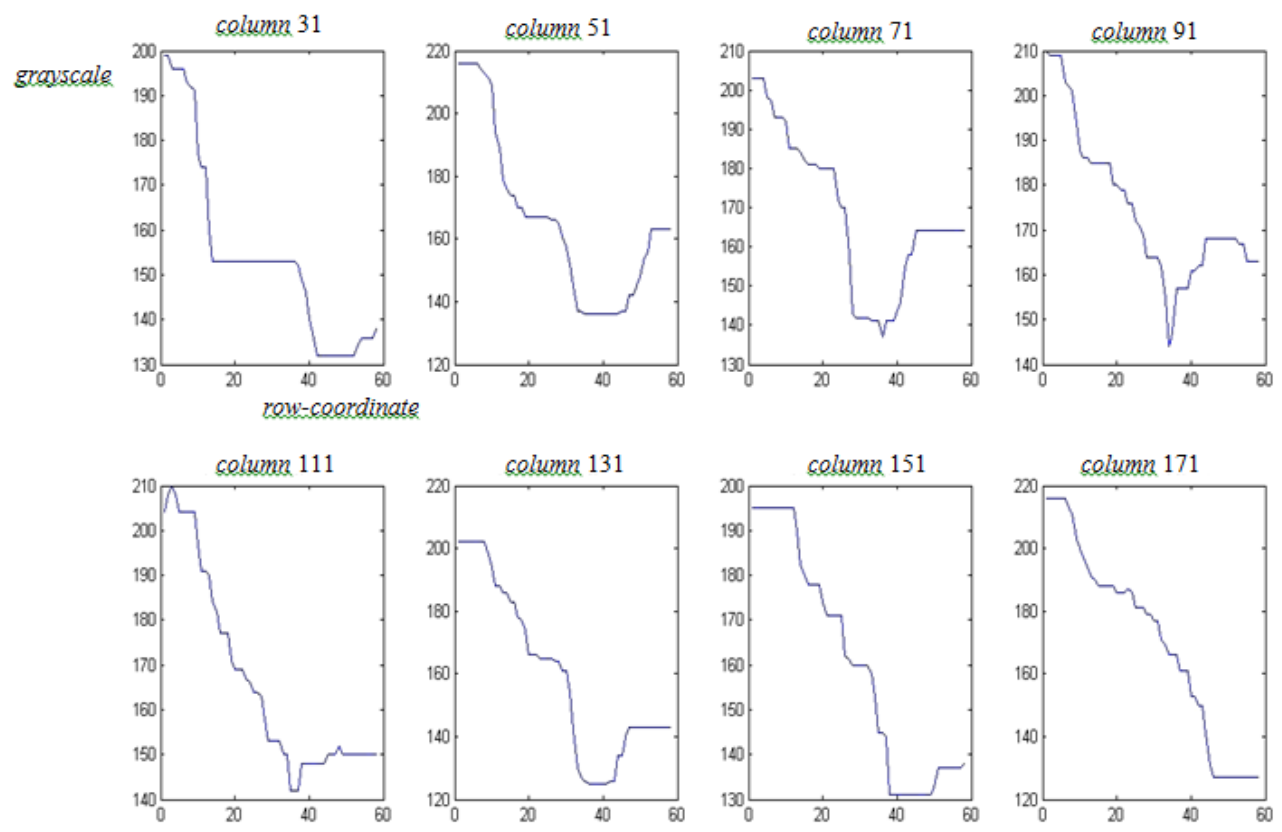

\section{Figure 6. Grayscale Value Distribution for Eyelid Area}

Each column gray minimum point is often not uniqueness. It is assumed that the $j$ column detects $n$ grayscale minimum points $\left\{x_{1}, \cdots, x_{n}\right\}$, and the line coordinates of the eyelid edge points in the $j$ column is shown as follows:

$$
X_{b j}=\frac{\sum_{i=1}^{n} x_{i}}{n}
$$

According to the above method, the edge point of the eyelid is extracted in Figure 7.

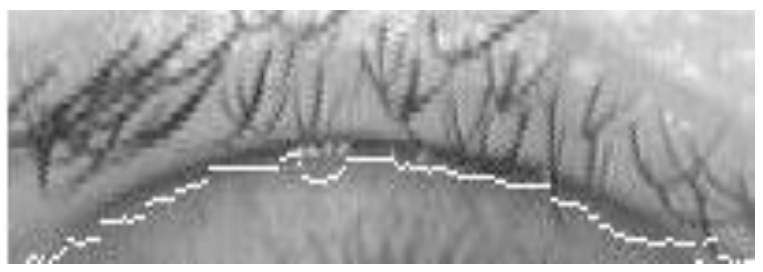

Figure 7. Edge Points on Eyelid

\subsection{Eyelid Edge Fitting}

Because of the influence of noise, the eyelid margin has discontinuous phenomena. To obtain the complete and smooth eyelid edge, the proposed algorithm uses the least squares fitting method to detect the edge points of the parabola fitting [10]. The parabolic equation is expressed as follows:

$$
x=a y^{2}+b y+c
$$

The formula (1) is transformed into the matrix form: 


$$
\begin{aligned}
& {\left[\begin{array}{c}
x_{1} \\
x_{2} \\
\vdots \\
x_{i} \\
\vdots \\
x_{n}
\end{array}\right]=\left[\begin{array}{ccc}
y_{1}^{2} & y_{1} & 1 \\
y_{2}^{2} & y_{2} & 1 \\
\vdots & \vdots & \vdots \\
y_{i}^{2} & y_{i} & 1 \\
\vdots & \vdots & \vdots \\
y_{n}^{2} & y_{n} & 1
\end{array}\right]\left[\begin{array}{l}
a \\
b \\
c
\end{array}\right]} \\
& \text { Assume } N=\left[\begin{array}{c}
x_{1} \\
x_{2} \\
\vdots \\
x_{i} \\
\vdots \\
x_{n}
\end{array}\right], Q=\left[\begin{array}{l}
a \\
b \\
c
\end{array}\right], P=\left[\begin{array}{ccc}
y_{1}^{2} & y_{1} & 1 \\
y_{2}^{2} & y_{2} & 1 \\
\vdots & \vdots & \vdots \\
y_{i}^{2} & y_{i} & 1 \\
\vdots & \vdots & \vdots \\
y_{n}^{2} & y_{n} & 1
\end{array}\right]
\end{aligned}
$$

The least square fitting method is employed to do the parabolic fitting to the edge points of eyelid, and the least squares solution of the formula (4) is shown as follows:

$$
Q=\left(P^{H} P\right)^{-1} P^{H} N
$$

The coordinates $\left(x_{i}, y_{i}\right)$ of the detected edge points are fed into the formula (5), and $Q$ is calculated to fit out the edge of the eyelid, and the results are shown in Figure 8.

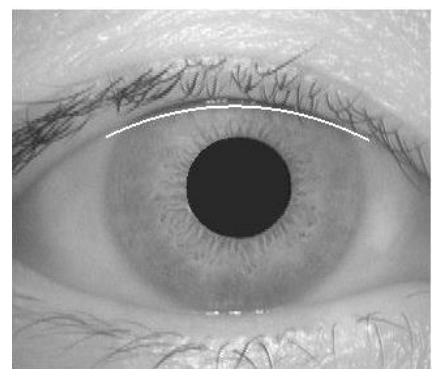

\section{Figure 8. Eyelid Border Fitting}

\section{The Precise Localization of the Eyelid}

Because the grayscale morphological operations make the edge transition zone wider, the edge of the eyelid fitting is not accurate, which need an accurate localization based on the eyelid fitting results. Compared with the iris, the edge of the eyelid is darker, and the grayscale value is small. Therefore, during the process of moving the detected eyelid parabolic up and down in the neighborhood, the pixel grayscale accumulating value of the parabola in eyelid edge will have jumping change. According to the position of the maximum value of jumping change, the edge of the eyelid can be localization accurately. In the parabolic equation $x=a y^{2}+b y+c, c$ is regarded as the parameters to decide the updown location of the parabola. The true eyelid edge position parameter $c_{0}$ should satisfy the following formula:

$$
c_{0}=\arg \max _{\text {curve }(c)}\left|\frac{\partial}{\partial c} \int_{\text {curve }(c)} I(x, y) d s\right|
$$

By the integral of the pixel grayscale value on the parabolic and $c$ seeking for difference, the formula (6) output can reach a maximum $\mathrm{c}$ as $\mathrm{c} 0 \mathrm{c} 1$ is set as the parameter of the eyelid fitting parabolic equation. The parameter parabolic equation $\mathrm{c}$ up-down 
moving range $[\mathrm{c} 1-15, \mathrm{c} 1+15]$. The final result of the eyelid precise localization is shown in Figure 9.

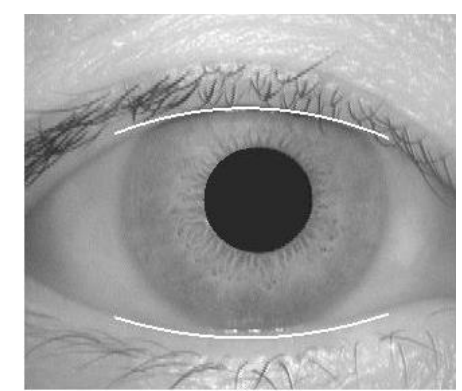

Figure 9. Eyelid Precise Localization

\section{The Results and Analysis of the Experiments}

In order to test the performance of the algorithm, each human eye is chosen as 1 sample image from 108 eye images in CASIA V1.0 iris database [11], and there are a total of 108 eye images. The eyelid detection algorithm and hough algorithm are compared from the subjective accuracy [9], and time consuming, which is shown in Table1.

Table 1. Performance Comparison between the Proposed Algorithm and Hough Transform

\begin{tabular}{ccccc}
\hline $\begin{array}{c}\text { Algorithm } \\
\text { slowest time /s }\end{array}$ & Subjective accuracy/\% & Average time /s & The fastest time /s & The \\
\hline Hough transform & 92.2 & 5.64 & 4.9 & 6.89 \\
The proposed algorithm & 93.5 & 1.43 & 1.28 & 1.98 \\
\hline
\end{tabular}

Some eyelid localization example by the proposed algorithm and hough transform is shown in Figure 10 and Figure 11.

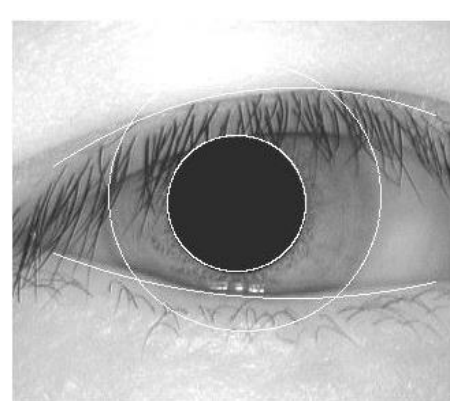

(a) Hough Transform

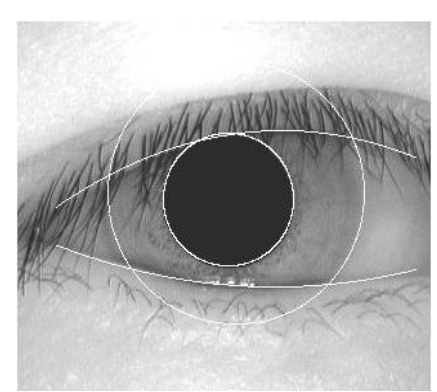

(b) The Proposed Algorithm

Figure 10. Eyelid Detection Result (CISIA V1.0) 


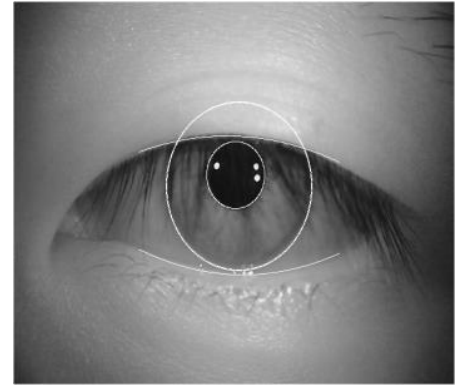

(a) Hough Transform

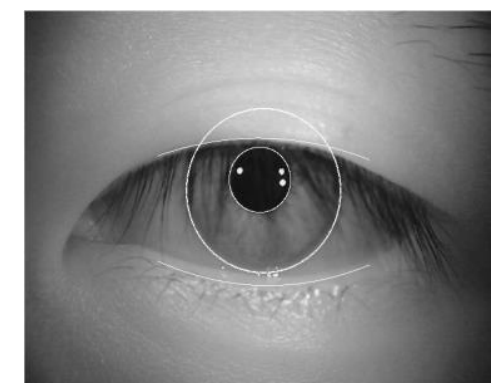

(b) The Proposed Algorithm

\section{Figure 11. Eyelid Detection Result (CISIA V2.0)}

\section{Conclusion}

Eyelid detection has an important significance to improve the rate of the iris recognition. A kind of fast and effective detection algorithm is put forward to localize the eyelids. The grayscale characteristics of the eyelid edge are used to localize the edge points. The least square method is used for fitting to the edges of the eyelids. The edge of the eyelid is located precisely. The eyelid detection algorithm reduces the threedimensional search space of the traditional algorithm to one-dimensional one to obviously improve the search speed. In the future, the work direction is to look for the adaptive structure element to better remove the upper eyelid eyelashes interference in gray scale morphological operations.

\section{References}

[1] Q. C. Tian and R. S. Zhang, "The biological feature recognition review", Application Research of computers, vol. 26, no. 12, (2009), pp. 4404-4407.

[2] V. Dorairaj, N. Schmid and G. Fahmy, "Performance evaluation of non-ideal iris based recognition system implementing global ICA encoding image processing”, IEEE Int. Conf., Italy, (2005).

[3] Y. Lai, C. H. Lu and C. Y. Lu, "The occlusions detection of iris recognition for eyelid and eyelash", Journal of Computer Aided Design \& computer graphics, vol. 19, no. 3, (2007), pp. 346-348.

[4] B. J. Kang and K. P. Park, "A robust eyelash detection based on iris focus assessment", Pattern Recognition, vol. 28, (2007), pp. 1630-1639.

[5] R. Wildes, "Iris recognition: an emerging biometric technology", Proceedings of the IEEE, vol. 85, no. 9, (2010), pp. 1348-1363.

[6] L. Masek, "Recognition of human iris patterns for biometricidentification", School of Computer Science and Software Engineering, University of Western Australia, Australia, (2003).

[7] M. Li, T. N. Tan and Y. H. Wang, "Personal identification based on iris texture analysis", IEEE Transactions on Pattern Analysis and Machine Intelligence, vol. 25, no. 12, (2011), pp. 1519-1533.

[8] W. Kong and D. Zhang, "Accurate iris segmentation based on novel reflection and eyelash detection model", Proceedings of 2001 International Symposium on Intelligent Multimedia, (2001), Hong Kong.

[9] Q. C. Tian, "Feature extraction and classification of imperfect localization of the iris segmentation", Xian: Northwestern Poly technical University Library, (2005).

[10] J. Zhou and T. Luo, "An iris location algorithm based on the geometric characteristics", Journal of the Institute of logistics engineering, vol. 26, no. 3, (2012), pp. 81-85.

[11] "Institute of Automation", Chinese Academy of Sciences, CASIA iris image database (version 1.0) [DB/OL].[2010-11-02]. http: / /www. Sinobiometrics. com.2004. 


\section{Authors}

Pengfei Cai, received his Master Degree from Zhengzhou University in 2010. He is now a experimentalist of Henan Mechanical and Electrical Engineering College in China. his research interests include software engineering, pattern recognition, data mining. He has published more than 4 papers on journals and conferences.

Chongke Wang, received his Master Degree from Wuhan University Of Technology in 2009. He is now an experimentalist of Henan Mechanical and Electrical Engineering College in China. His research interests include software engineering, data mining. He has published more than 5 papers on journals and conferences. 